\title{
Time (in)dependence in general relativity
}

\author{
S. Deser ${ }^{\mathrm{a})}$ \\ Department of Physics, Brandeis University, Waltham, Massachusetts 02454 \\ and Lauritsen Laboratory, California Institute of Technology, Pasadena, California 91125 \\ J. Franklin ${ }^{\text {b) }}$ \\ Department of Physics, Reed College, Portland, Oregon 97202
}

(Received 6 July 2006; accepted 1 December 2006)

\begin{abstract}
We clarify the conditions for Birkhoff's theorem, that is, time independence in general relativity. We work primarily at the linearized level where guidance from electrodynamics is particularly useful. As a bonus, we also review how the equivalence principle results from general relativity. The basic time-independent solutions due to Schwarzschild and Kerr provide concrete illustrations of the theorem. Only familiarity with Maxwell's equations and tensor analysis is required. (C) 2007 American Association of Physics Teachers.
\end{abstract}

[DOI: 10.1119/1.2426351]

\section{INTRODUCTION}

A major obstacle to teaching general relativity is the initially confusing mathematics underlying useful, physical simplifications. We focus in this paper on the conditions that lead to the simplest regime, time independence. Because general relativity is coordinate invariant, what does it mean to speak of a particular coordinate's absence? The answer is yes (and is our one excursion into the higher mathematics). The answer is illuminating. Loosely, we expect that there exists a choice of coordinate frame in which the gravitational field does not depend on $t$. But is this a meaningful, that is, invariant, criterion? The answer is yes: it means that the spacetime geometry allows the existence of a Killing vector field $f_{\mu}(x)$ that obeys the tensor equation

$$
\begin{aligned}
D_{\nu} f_{\mu}+D_{\mu} f_{\nu} \equiv & \partial_{\nu} f_{\mu}+\partial_{\mu} f_{\nu}-g= \\
& \times\left(\partial_{\nu} g_{\mu \rho}+\partial_{\mu} g_{\nu \rho}-\partial_{\rho} g_{\mu \nu}\right) f_{\sigma}=0,
\end{aligned}
$$

where $g_{\mu \nu}$ is the metric and $D_{\mu}$ is the covariant derivative with respect to it, as defined in Eq. (1). We use the signature $(-+++)$ and units such that $c=1$. If $f_{\mu}$ is also timelike $\left(f^{2}\right.$ $<0$ ), then the solution in the frame where $f_{\mu}=g_{0 \mu}$ (more manifestly, the contravariant form $f^{\mu}$ of the vector is $f^{\mu}$ $=\delta_{0}^{\mu}$ ) implies that

$$
\partial_{0} g_{\mu \nu}=0
$$

and there is no time dependence. [A special property of timeindependent geometries is that in (and only in) them, matter systems such as particles retain a conserved energy, just as in flat space.]

The main point of the above discussion is to have reexpressed the issue of when a given geometry is time independent, that is, when there exists a frame where Eq. (2) holds, as a covariant (coordinate-independent) criterion: the existence of solutions to Eq. (1). All this transcription makes no reference to field equations. There exist many frames where $t$ dependence is present, but that is not the point. Finally, it is, of course, totally false that every geometry has a static frame-the Killing equation is a strong requirement.

\section{MAXWELL}

We begin with electrodynamics, whose field equations outside sources, unlike general relativity, can be written entirely in terms of gauge invariant field strengths,

$$
\begin{aligned}
& \nabla \cdot \mathbf{E}=0, \\
& \nabla \cdot \mathbf{B}=0, \\
& \dot{\mathbf{E}}=\nabla \times \mathbf{B}, \\
& \dot{\mathbf{B}}=-\nabla \times \mathbf{E} .
\end{aligned}
$$

The $\dot{\mathbf{E}}$ equation's longitudinal part (see the following) implies that $\dot{\mathbf{E}}^{L}=0$, which exhibits the fact that the "Coulomb" part of $\mathbf{E}$ is always time independent, whatever the behavior of the interior charges. The remaining, dynamical transverse part $\mathbf{E}^{T}$ and its partner $\mathbf{B}$ (transverse by definition) cannot depend on time if they vanish identically, which is the case for spherically symmetric configurations: any $\mathbf{E}(r)$ is necessarily of the form $\nabla S(r)$ and is purely longitudinal. There is no monopole radiation; it is also the only guaranteed static case, as dipole and higher configurations define transverse vectors. Equation (3) does not thereby require time dependence, or electro/magneto-statics would not exist.

We recall that the transverse/longitudinal division of any vector field $\mathbf{V}$ is a decomposition of unity,

$$
V_{i}=\left[\left(\delta_{i j}-\hat{k}_{i} \hat{k}_{j}\right)+\hat{k}_{i} \hat{k}_{j}\right] V_{j},
$$

along some arbitrary unit vector direction $\hat{k}$. Its more familiar Fourier transform is

$$
\mathbf{V}=\mathbf{V}^{T}+\mathbf{V}^{L}
$$

where $\nabla \cdot \mathbf{V}^{T}=\nabla \times \mathbf{V}^{L}=0$.

Our discussion has been couched in terms of the gauge invariant field strengths $\mathbf{E}$ and $\mathbf{B}$, whose time (in-)dependence is unaffected by the choice of gauge. The underlying potentials $\left(A_{0}, \mathbf{A}\right)$ are another story: even if 
$(\mathbf{E}, \mathbf{B})$ are static, there exist gauge choices for which the potentials do depend on $t$ by adding gauge terms $\partial_{\mu} \Lambda(r, t)$ that do not affect $F_{\mu \nu}=\partial_{\mu} A_{\nu}-\partial_{\nu} A_{\mu}$. In any case the transverse vector potentials are unaffected, being gauge invariant. Only $\left(A_{0}, \mathbf{A}^{L}\right)$ can be altered, keeping $\mathbf{E}^{L}$ unchanged.

Armed with the vector field analysis, we proceed to the quite analogous spin 2 , tensor, case.

\section{GENERAL RELATIVITY}

For our purposes the gravitational field is a glorified tensor version of the vector Maxwell field $A_{\mu}$, and we expect similar properties of the results there to apply. At the linearized level, the Einstein equations outside sources are

$$
\begin{aligned}
2 G_{\mu \nu} \equiv & \square h_{\mu \nu}-\left(\partial_{\mu} \partial_{\alpha} h_{\nu}^{\alpha}+\partial_{\nu} \partial_{\alpha} h_{\mu}^{\alpha}\right)+\partial_{\mu} \partial_{\nu} h \\
& -\eta_{\mu \nu}\left(\square h-\partial_{\alpha} \partial_{\beta} h^{\alpha \beta}\right)=0
\end{aligned}
$$

for the field $h_{\mu \nu}$ with $h \equiv h_{\alpha}^{\alpha}$; all indices are moved by the Minkowski metric $\eta_{\mu \nu}$. As for Maxwell's equations, we decompose Eq. (6) into space and time components, with the simplifying notation $h_{0 i} \equiv N_{i}$ and $h_{00} \equiv N$. The theory is invariant under linearized gauge/coordinate transformations $h_{\mu \nu} \rightarrow h_{\mu \nu}+\partial_{\mu} \xi_{\nu}+\partial_{\nu} \xi_{\mu}$, that is, $G_{\mu \nu}\left(\partial_{\mu} \xi_{\nu}+\partial_{\nu} \xi_{\mu}\right)=0$, an invariance that is useful to exploit.

The component form (the linearized version of a decomposition used long ago to analyze the full theory ${ }^{1}$ ) of Eq. (6) is

$$
\begin{aligned}
2 G_{00}= & \nabla^{2} \tilde{h}-\partial_{i} \partial_{j} h_{i j}, \\
2 G_{0 i}= & \nabla^{2} N_{i}-\partial_{j} \dot{h}_{j i}-\partial_{i} \partial_{j} N_{j}+\partial_{i} \dot{\tilde{h}}, \\
2 G_{i j}= & \square h_{i j}+\partial_{i} \dot{N}_{j}+\partial_{j} \dot{N}_{i}-\left(\partial_{i} \partial_{k} h_{k j}+\partial_{j} \partial_{k} h_{k i}\right)+\left(\delta_{i j} \nabla^{2}\right. \\
& \left.-\partial_{i} \partial_{j}\right)(N-\widetilde{h})+\ddot{\widetilde{h}} \delta_{i j}+\delta_{i j}\left(\partial_{m} \partial_{n} h_{m n}-2 \partial_{k} \dot{N}_{k}\right),
\end{aligned}
$$

with $\tilde{h} \equiv h_{i}^{i}$ the trace of the spatial part of the field. This slightly complicated set of equations simplifies when we decompose the spatial tensors $h_{i j}$ and the vectors $N_{i}$, the latter into transverse/longitudinal parts via Eq. (4), the former by the following partition of unity:

$$
\begin{aligned}
& h_{i j}=h_{i j}^{T T}+h_{i j}^{T}+\partial_{i} h_{j}+\partial_{j} h_{i}, \\
& \partial_{i} h_{i j}^{T T}=\partial_{i} h_{i j}^{T}=0=h_{i i}^{T T}, \\
& h_{i j}^{T}=\frac{1}{2}\left(\delta_{i j}-\nabla^{-2} \partial_{i} \partial_{j}\right) h^{T} .
\end{aligned}
$$

The six components of $h_{i j}$ are decomposed linearly, orthogonally, and uniquely into two TT (transverse traceless), one T (traceless), and three $h_{i}$ parts. Any spatial tensor equation thus consists of three independent sets. The four quantities $\left(h_{i}, N_{i}^{L}\right)$ are pure gauges (variables that can be arbitrarily changed by using the gauge freedom of the theory) that cry out to be set to zero, leaving the gauge invariant set $\left(h_{i j}^{T T}, h^{T}, N_{i}^{T}, N\right)$ once we use the available gauge invariance. Now Eq. (7) reduces to

$$
\begin{aligned}
& 2 G_{00}=\nabla^{2} h^{T}=0, \\
& 2 G_{0 i}=\nabla^{2} N_{i}^{T}+\partial_{i} \dot{h}^{T}=0,
\end{aligned}
$$

$$
\begin{aligned}
2 G_{i j}= & \square h_{i j}^{T T}+\left(\partial_{i} \dot{N}_{j}^{T}+\partial_{j} \dot{N}_{i}^{T}\right)+\left(\delta_{i j} \nabla^{2}-\partial_{i} \partial_{j}\right)\left(N-\frac{1}{2} h^{T}\right) \\
& +\frac{1}{2}\left(\delta_{i j}+\nabla^{-2} \partial_{i} \partial_{j}\right) \ddot{h}^{T}=0 .
\end{aligned}
$$

The time independence of $h^{T}$ follows from the longitudinal part of Eq. (9b), and the relation $N=\frac{1}{2} h^{T}$ follows from Eq. $(9 c)$. This seemingly innocuous equality is none other than the expression of Einstein's principle of equivalence. This expression of the equivalence principle even applies to full GR. ${ }^{1}$ The latter states that (in suitable units) the inertial and gravitational masses of every physical system are equal. Inertial mass/energy is the conserved quantity that (in the linear regime) sums over the $T_{00}$ contributions of the interior sources. This sum is the monopole moment of the Poisson equation (9a) (if we restore $T_{00}$ as its right-hand side); hence it is the coefficient of the leading $1 / r$ term in $h^{T}$. In contrast, gravitational mass is a very different quantity that determines the system's gravitational pull, the "Newtonian" force, on slow particles. (Einstein implicitly assumed the existence of static frames, as we have also established here.) This force is the gradient of the leading, $1 / r$, part of $h_{00}$. Thus, in general relativity the field equation (9c) enforces the universal equality of the desired $1 / r$ coefficients.

The time independence of $N_{i}^{T}$ results from the transverse vector part of Eq. (9c): The four "Newtonian" components of the field are time-independent outside sources. Time dependence can reside only in the remaining $h_{i j}^{T T}$ dynamical modes, namely those field components unaffected by the choice of gauge and undetermined by the interior sources. Hence $t$ independence is forced whenever TT tensors are forbidden. Spherical symmetry is one such case, because all spherically symmetric tensors have the form

$$
S_{i j}(r)=\delta_{i j} A(r)+\partial_{i} \partial_{j} B(r),
$$

and so, by Eq. (8a), have no TT parts. This result is the basis of Birkhoff theorem: ${ }^{2}$ all spherically symmetric configurations are also time independent, a result valid also in full general relativity.

Unlike Maxwell, there is another category of fields lacking a TT part, namely those with dipole character. As we saw there, dipoles permit a transverse vector, but their single direction is not generic enough to construct a TT tensor. Axial symmetry does permit TT, for example via the tensor harmonic $P_{2}(\cos \theta)$. To summarize at this point, both Maxwell and linearized general relativity gauge fields only allow time dependence of their true dynamical excitations, and only when those modes can be present, which always excludes spherical symmetry and also dipole symmetry for the general relativity case.

\section{KERR AND SCHWARZSCHILD}

It is instructive, at the linearized level, to relate the exterior solution properties to explicit matter sources. In electrodynamics the current consists of two parts: the charge density $\rho$ and the longitudinal current $\mathbf{j}^{L}$, which obey the continuity equation $\dot{\rho}+\nabla \cdot \mathbf{j}^{L}=0$, and the transverse current $\mathbf{j}^{T}$. The $\left(\rho, \mathbf{j}^{L}\right)$ subset couples only to the longitudinal electric field, which is equivalent to it, and, as we saw, is time independent away from sources. The transverse electric and magnetic fields are generated by the transverse current and can be time dependent if $\mathbf{j}^{T}$ is. Similar reasoning applies to general relativity: the source here is the tensor $T_{\mu \nu}$, whose $\left(T_{00}, T_{0 i}^{L}\right)$ 
components are like $\left(\rho, \mathbf{j}^{L}\right)$. They obey the same continuity equation and excite only the metric component $h^{T}$, which is also $t$ independent outside of source distributions. Because general relativity is a tensor theory, there is another "charge" associated with momentum like $T_{00}$ was with energy, namely $\left(T_{0 i}, T_{i j}^{L}\right)$,which also obeys continuity and is coupled to $N_{i}^{T}$. The remaining source part, $T_{i j}^{T T}$, which may, but need not, depend on time, excites the dynamical $h_{i j}^{T T}$ fields.

An important example of time independence is furnished by the Kerr solution ${ }^{3,4}$ of full general relativity, which we will reproduce in the following. In our linearized context, the static metric is generated by a time-independent spinning point mass with

$$
T_{00}=m \delta^{3}(\mathbf{r}), \quad T_{0 i}=a m \epsilon_{i j k} s_{j} \partial_{k} \delta^{3}(\mathbf{r}),
$$

where $s_{j}$ denotes the (constant) unit spin vector. As explained, for example, in Ref. 5, the space integral of $T_{00}$ is the total mass $m$, and that of $T_{0 i}$ vanishes because there is no momentum. Its first moment, the angular momentum $\mathbf{J}$, is given by $\mathbf{J}=a m \mathbf{s}$. The notation choice that expresses $J$ $\sim a m$ is historical, but has the virtue that $m=0$ is actually just flat space (also in full general relativity). ${ }^{7}$ The opposite limit, $a=0$, defines the spherically symmetric static Schwarzschild solution.

We will not discuss in detail the full general relativity extensions of our linear results. Consider, without deriving it (there is no simple way to do so), the full Kerr interval

$$
d s^{2}=-g_{t t} d t^{2}+g_{r r} d r^{2}+g_{\theta \theta} d \theta^{2}+g_{\phi \phi} d \phi^{2}+2 g_{t \phi} d t d \phi .
$$

There are five functions of $(r, \theta)$, which are (in units of $c$ $=1=16 \pi G$ )

$$
\begin{aligned}
g_{t t} & =-\left(1-2 M r / \rho^{2}\right), \\
g_{r r} & =\rho^{2} / \Delta, \\
g_{\theta \theta} & =\rho^{2}, \\
g_{\phi \phi} & =\sin ^{2} \theta\left[\left(r^{2}+a^{2}\right)+2 a^{2} M r \sin ^{2} \theta / \rho^{2}\right], \\
g_{t \phi} & =-2 a M r \sin ^{2} \theta / \rho^{2}, \\
\text { with } \rho^{2} & \equiv r^{2}+a^{2} \cos ^{2} \theta \text { and } \Delta \equiv a^{2}-2 M r+r^{2} .
\end{aligned}
$$

The linearized limit of Eqs. (12) and (13), or equivalently its asymptotic form, is a superposition of the (linearized) Schwarzschild solution and a spin term $h_{0 \phi}$ corresponding to the source (11):

$$
h_{00}=\frac{2 m}{r},
$$

$$
\begin{aligned}
& h_{0 \phi}=-\frac{2 a m \sin ^{2} \theta}{r}, \\
& h_{i j}=\frac{2 m}{r} \frac{x_{i} x_{j}}{r^{2}} .
\end{aligned}
$$

We emphasize that the time independence here is derivable directly from the exterior equations, apart from details of the interior source, as we would expect for a spinning spherical ball of charge in E\&M, its natural analog.

\section{CONCLUSIONS}

By working primarily in the linearized limit, we have provided, using the Maxwell template, a framework for understanding the basis of time independence in general relativity in terms of the underlying physics and source geometry. Our main conclusion is that the time dependence of solutions of gauge theories such as Maxwell's or general relativity is a property of their radiation modes. If these are forbidden due to spherical (dipole) symmetry, then time independence is guaranteed. In particular, the Kerr and Schwarzschild solutions illustrate the absence of dipole and monopole excitations. Although the full general relativity is unavoidably more complicated (and involves global issues we have bypassed here), our results capture at least its long distance properties.

\section{ACKNOWLEDGMENTS}

We are grateful to Professor J. Hartle for stimulating criticism that led to this (we hope) improved version of our earlier paper. This work was supported by NSF Grant No. PHY04-01667.

\footnotetext{
${ }^{a}$ Electronic address: alvaro.gonzalez@unizar.es

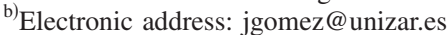

${ }^{1}$ R. Arnowitt, S. Deser, and C. W. Misner, "The dynamics of general relativity," in Gravitation: An Introduction to Current Research, edited by L. Witten (Wiley, New York, 1962) (reprinted as gr-qc/0405109).

${ }^{2}$ For the history and a modern derivation, see S. Deser and J. Franklin, "Schwarzschild and Birkhoff a la Weyl," Am. J. Phys. 73(3), 261-264 (2005) (gr-qc/0408067).

${ }^{3}$ Roy P. Kerr, "Gravitational field of a spinning mass as an example of algebraically special metrics," Phys. Rev. Lett. 11(5), 237-238 (1963).

${ }^{4}$ Robert H. Boyer and Richard W. Lindquist, "Maximal analytic extension of the Kerr metric," J. Math. Phys. 8(2), 265-281 (1967).

${ }^{5}$ Charles W. Misner, Kip S. Thorne, and John Archibald Wheeler, Gravitation (Freeman, New York, 1973).

${ }^{6}$ J. Franklin and P. T. Baker, "Linearized Kerr and spinning massive bodies: An electrodynamics analogy," Am. J. Phys. (to be published).

${ }^{7}$ The transformation that takes us back to Cartesian coordinates in this case is: $x=\sqrt{r^{2}+a^{2}} \sin \theta \cos \phi, y=\sqrt{r^{2}+a^{2}} \sin \theta \sin \phi$, and $z=r \cos \theta$.
} 


\title{
Nodes of wavefunctions
}

\author{
M. Moriconi \\ Departamento de Física, Universidade Federal Fluminense, Av. Litorânea s/n, Boa Viagem - CEP \\ 24210-340, Niterói, Rio de Janeiro, Brazil
}

(Received 10 July 2006; accepted 10 November 2006)

\begin{abstract}
We give a simple argument to show that the $n$th wavefunction for the one-dimensional Schrödinger equation has $n-1$ nodes. We also show that if $n_{1}<n_{2}$, then between two consecutive zeros of $\psi_{n_{1}}$, there is a zero of $\psi_{n_{2}}$. (C) 2007 American Association of Physics Teachers.

[DOI: $10.1119 / 1.2404960]$
\end{abstract}

The time-independent Schrödinger equation in one dimension is a linear, second-order differential equation, which in units where $\hbar^{2} / 2 m=1$, can be expressed as

$$
-\psi^{\prime \prime}+V \psi=E \psi
$$

All the properties of the wavefunction are encoded in Eq. (1). We would like to know the properties of the zeros of its eigenfunctions, that is, how many nodes there are for the wavefunction $\psi_{n}$ corresponding to the $n$th energy level. Arguments based on the variational principle ${ }^{1,2}$ show that the ground state wavefunction has no nodes, but do not say much about wavefunctions for higher energy levels. This question is considered too detailed ${ }^{3,4}$ and is usually discussed in the more mathematical literature such as Refs. 5-7. The number of zeros possessed by a solution of a linear, secondorder, differential equation and their distribution is the subject of Sturm-Liouville theory. ${ }^{6}$ Two of the main theorems in Sturm-Liouville theory are the separation and the comparison theorems. For the Schrödinger equation, the separation theorem states that the zeros of two linearly independent solutions of Eq. (1) alternate; the comparison theorem states that if $n_{1}<n_{2}$, then between two consecutive zeros of $\psi_{n_{1}}$ there is a zero of $\psi_{n_{2}}$. There are stronger results that show that the solution corresponding to the $n$th eigenvalue has precisely $n-1$ zeros, and, if the potential goes to infinity as $|x| \rightarrow \infty$, then the eigenvalues form a discrete unbounded sequence. These theorems are very important in quantum mechanics, but they are usually not discussed in quantum mechanics textbooks.

The purpose of this note is to provide a simple, intuitive, argument for some of these results. We show that $\psi_{n}$ has $n$ -1 nodes, ${ }^{8}$ and that if $n_{2}>n_{1}$, then between two zeros of $\psi_{n_{1}}$, there is a zero of $\psi_{n_{2}}$. The second result is simpler to prove, and we include it here for completeness. The first result is more difficult to prove and is the main contribution of this paper. The node structure of wavefunctions is important. For example, the fact that the ground state function has no nodes was one of the ingredients Feynman used in his theory of liquid helium. ${ }^{1}$ In the following we assume that the normalizable solutions of Eq. (1) exist, and that they are real, which is always true for the time-independent Schrödinger equation in one dimension.

Given a potential $V(x)$ we construct a new family of potentials $V_{a}(x)$, such that $V_{a}(x)=V(x)$ for $-a<x<a$, and $V(x)=\infty$ for $|x|>a$. For $a=\epsilon$ sufficiently small we have an infinite potential well and the wave functions are well known: $\psi_{k}^{(\mathrm{o})}(x ; \epsilon) \propto \sin (k \pi x / \epsilon), \quad k=1,2, \ldots$, and $\psi_{k}^{(\mathrm{e})}(x ; \epsilon)$ $\propto \cos ((2 k+1) \pi x / 2 \epsilon), k=0,1,2, \ldots$, where the superscripts (e) and (o) refer to the even/odd parity of the wavefunctions. Clearly, $\psi_{n}(x ; \epsilon)$ has $n-1$ nodes between $-\epsilon$ and $\epsilon$ where it vanishes. We first focus on the ground state wave function, which we may assume to be positive, because there are no nodes, that is, $\psi_{1}^{\prime}(-\epsilon ; \epsilon)>0$ and $\psi_{1}^{\prime}(\epsilon ; \epsilon)<0 .{ }^{9}$ Imagine that we separate the "walls" by increasing $a$. The wavefunction $\psi_{1}(x ; a)$ will become a better and better approximation to the true ground state wave function, starting from a wavefunction without any nodes. Can this wavefunction develop a node between $\pm a$, for some value of $a$ ? If so, there are two possibilities: either (1) at least one of the derivatives at $\pm a$ must change sign or (2) the derivatives at $\pm a$ do not change sign, but the wavefunction develops two zeros through its decrease at some point between $-a$ and $a$. In both cases there will be a critical value of $a$ such that the wavefunction and its first derivative vanish at the same point. In case (1) the wavefunction and its derivative vanish at one of the boundary points, because the boundary condition implies that the wavefunction vanishes at the boundaries, and if the derivative changes sign, then it must be zero for the critical value of $a$. In case (2) there must be a value of $a$ such that the wavefunction touches the real axis just before the wavefunction dips down and develops two zeros, and therefore has zero value and zero slope there. Because the Schrödinger equation is a linear, second-order, ordinary differential equation, it has a unique solution, given the value of the function and its derivative at the same point. But if the solution and its first derivative are zero at the same point, we conclude that the wavefunction must be identically zero, because $\psi=0$ satisfies the differential equations and the vanishing conditions of the function and its derivative. Because we assume that there is always a nontrivial solution of the Schrödinger equation for any value of $a$, we conclude that in both cases the ground state function can develop no nodes. The same reasoning shows that because the $n$th wavefunction starts with $n-1$ nodes, their number cannot increase nor decrease, because to develop a new zero or lose a zero, the wavefunction must go through one of the stages described in cases (1) and (2) as $a$ increases.

There are cases where the potential can support only a finite number of bound states, say $N$. What happens to the wavefunctions for $n>N$ ? As we separate the walls, some of the wavefunctions go to zero because the separation between the zeros increases as $a \rightarrow \infty$, and the positive energy states 
become the continuum spectrum. As an example, consider the potential well with two walls, as discussed in Ref. 10.

It is straightforward to show that between two consecutive zeros of $\psi_{n_{1}}$, there is a zero of $\psi_{n_{2}}$ for any $n_{2}>n_{1}$. This statement can be proved as follows. From the Schrödinger equation for $\psi_{n_{1}}$ and $\psi_{n_{2}}$, we see that

$$
\left(\psi_{n_{1}}^{\prime} \psi_{n_{2}}-\psi_{n_{2}}^{\prime} \psi_{n_{1}}\right)^{\prime}=\left(E_{n_{2}}-E_{n_{1}}\right) \psi_{n_{1}} \psi_{n_{2}}
$$

If $\psi_{n_{1}}$ has two consecutive zeros at $x_{1}$ and $x_{2}$, we may assume that $\psi_{n_{1}}$ is positive in the interval between the two zeros. If $\psi_{n_{2}}$ does not vanish in this interval, we may also assume it is positive. If we integrate Eq. (2) from $x_{1}$ to $x_{2}$ and note that $\psi_{n_{1}}^{\prime}\left(x_{1}\right)>0$ and $\psi_{n_{1}}^{\prime}\left(x_{2}\right)<0$, we obtain

$$
\begin{aligned}
& \psi_{n_{1}}^{\prime}\left(x_{2}\right) \psi_{n_{2}}\left(x_{2}\right)-\psi_{n_{1}}^{\prime}\left(x_{1}\right) \psi_{n_{2}}\left(x_{1}\right) \\
& =\left(E_{n_{2}}-E_{n_{1}}\right) \int_{x_{1}}^{x_{2}} d x \psi_{n_{1}}(x) \psi_{n_{2}}(x) .
\end{aligned}
$$

Because the left-hand side is negative definite and the righthand side is positive definite, we arrive at a contradiction. Therefore $\psi_{n_{2}}$ has a zero between two consecutive zeros of $\psi_{n_{1}}$.

The method presented here can be used to establish that the ground state wavefunction has no nodes in dimensions higher than one. All that is necessary is to consider an infinite potential well around the origin and separate the walls.
Because the ground state wavefunction starts with no nodes, it can develop no nodal lines, for reasons similar to those we have discussed.

\section{ACKNOWLEDGMENTS}

I would like to thank L. Moriconi and C. Tomei for useful discussions. The remarks of two anonymous referees are gratefully acknowledged, especially for pointing out Ref. 7 and correcting the wavefunctions for the infinite potential well. This work has been partially supported by Faperj.

${ }^{1}$ R. P. Feynman, Statistical Mechanics: A Set of Lectures (AddisonWesley, Reading, MA, 1998). See also Appendix A in the Ph.D. thesis by M. Cohen, "The energy spectrum of the excitations in liquid helium," 〈http://etd.caltech.edu/etd/available/etd-03192004-153651/〉.

${ }^{2}$ J. Mur-Petit, A. Polls, and F. Mazzanti, "The variational principle and simple properties of the ground-state wave function," Am. J. Phys. 70(8), 808-810 (2002).

${ }^{3}$ L. D. Landau and E. M. Lifschitz, Quantum Mechanics: Non-Relativistic Theory (Butterworth-Heinemann, Oxford, 1981).

${ }^{4}$ A. Galindo and P. Pascual, Quantum Mechanics (Springer, Berlin, 1991), Vol. 1.

${ }^{5}$ R. Courant and D. Hilbert, Methods of Mathematical Physics (WileyInterscience, New York, 1989), Vol. 1.

${ }^{6}$ P. Hartman, Ordinary Differential Equations (Wiley, New York, 1964).

${ }^{7}$ Géza Makay, "A simple proof for Sturm's separation theorem," Am. Math. Monthly 99, 218-219 (1992).

${ }^{8}$ We will call the ground state function $\psi_{1}$ instead of the more common notation $\psi_{0}$.

${ }^{9}$ The prime denotes the spatial derivative.

${ }^{10}$ S. Flügge, Practical Quantum Mechanics (Springer, New York, 1998), Problems 25 and 26. 


\section{Erratum: "The occupation of a box as a toy model for the seismic cycle of a fault" [Am. J. Phys. 73 (10), 946-952 (2005)]}

Álvaro González ${ }^{\mathrm{a})}$ and Javier B. Gómez ${ }^{\mathrm{b})}$

Departamento de Ciencias de la Tierra, Universidad de Zaragoza, C. Pedro Cerbuna 12, 50009 Zaragoza, Spain

Amalio F. Pacheco ${ }^{\text {) }}$

Departamento de Física Teórica and BIFI, Universidad de Zaragoza, C. Pedro Cerbuna 12, 50009 Zaragoza, Spain

(Received 20 November 2006; accepted 1 December 2006)

[DOI: $10.1119 / 1.2426356]$

The values of $f_{e}$ plotted in Fig. 6 and used to estimate $n^{*}$ were determined by a Monte Carlo simulation of the model instead of using Eq. (20). If, at a given time step in the simulation, the "alarm" was sounded and the model earthquake occurred, the latter was deemed as successfully forecasted. This assumption is incorrect, and leads to a value of $f_{e}$ smaller than the true one in Eq. (20). Given that $n$ is the number of time steps before sounding the alarm, if the earthquake occurs at the $n$th time step, the alarm has still not been sounded, and the earthquake should be considered a prediction failure. An earthquake in the box model cannot occur before the $N$ th time step of each cycle, so $f_{e}=0$ if and only if $n<N$. This error caused $f_{e}=0$ also for $n=N$.

We give here a revised version of Fig. 6. The correct values of $f_{e}$ and $L=f_{a}+f_{e}$ are only slightly higher than those previously published. This correction changes the value of $n^{*}$ (19 time steps, instead of 20). It also modifies the results in Eq. (22),

$$
f_{a}\left(n^{*}\right)=0.432, \quad f_{e}\left(n^{*}\right)=0.084, \quad L\left(n^{*}\right)=0.516,
$$

and the value in Eq. (23) for the Parkfield sequence:

$$
\tau n^{*}=14.1 \mathrm{yr} \text {. }
$$

We apologize for this error and hope that this note will serve to clarify the convention for calculating $f_{e}$ with discrete probability distributions.

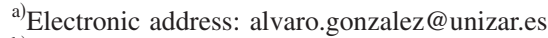

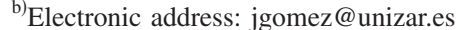

${ }^{c)}$ Electronic address: amalio@unizar.es
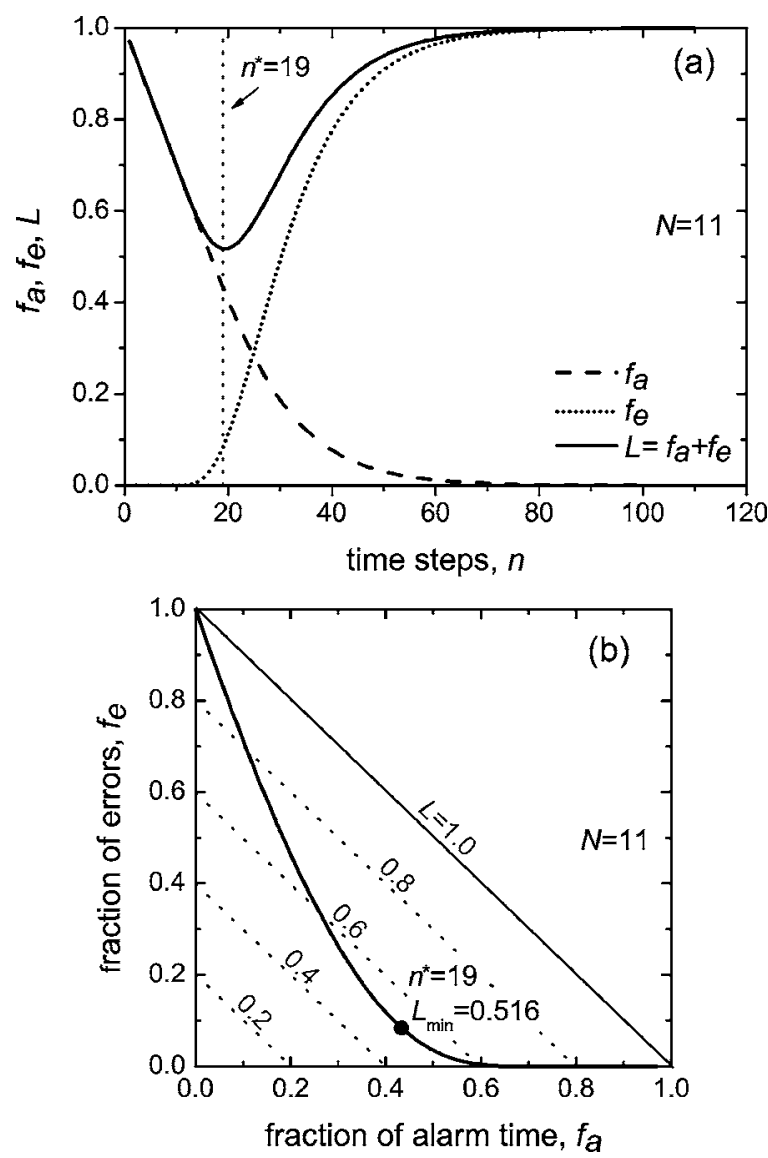

Fig. 6. Corrected version of Fig. 6 of the original article. 